\title{
Embarazo heterotópico: A propósito de un caso
}

\author{
Gustavo Gómez T.*; Carlos A. Agudelo**
}

La presencia simultánea de embarazo Ectópico y embarazo Intrauterino, es un evento Obstétrico relativamente raro. La incidencia inicial fue estimada entre 1:30.000 (1) y 1:50.000 (2). Sin embargo nuevos reportes han encontrado incidencia más alta: $1: 6.788$ (3) y 1:7.963 (4). El creciente índice de enfermedad pélvica inflamatoria (5), el uso de inductores de ovulación y las modernas técnicas de reproducción asistida contarían para dicha tendencia (6).

Aunque el embarazo Heterotópico continúa siendo una entidad rara, la condición debe ser considerada tanto por el clínico como por el ultrasonografista en aras de un diagnóstico preciso y un tratamiento adecuado.

\section{Reporte del caso}

\section{H.C. No. 1116196}

Paciente de 26 años G:4 P:2 A:1 con amenorrea no confiable de más o menos de 7 semanas quien consulta por dolor en el hemiabdomen inferior tipo "Cólico" y que cede con analgésicos. No refería sangrado vaginal ni otros síntomas.

A la valoración clínica se encuentra paciente con signos vitales estables. El examen abdominal no reveló signos de irritación peritoneal. La valoración pélvica no evidenció sangrado vaginal encontrándose ostium cervical cerrado. La valoración de las características uterinas fue difícil por defensa abdominal voluntaria. Había dolor marcado a la palpación de fondo de saco posterior.

La evaluación inicial incluyó ultrasonografía pélvica que reporta saco gestacional intrauterino con una longitud céfalo-caudal de $20 \mathrm{~mm}$ para embarazo de 9 semanas y un segundo saco gestacional, posiblemente extrauterino, con feto vivo y Biometría para más o menos 8 semanas. Figura 1.

La laparoscopia encontró un hemoperitoneo de más o menos $100 \mathrm{cc}$, embarazo ectópico ístmico-ampular derecho no roto y útero aumentado de tamaño.

La paciente fue llevada a la laparotomía. Se realizó salpingectomía, cuya patología reportó embarazo ectópico tubárico.

La paciente evolucionó satisfactoriamente y fue dada de alta a las 48 horas postquirúrgicas.

Al momento del reporte la paciente cursa con un embarazo de 26 semanas.

\section{Discusión}

El primer caso de embarazo heterotópico fue reportado por Duverney en 1708 como hallazgo de autopsia. Devoe y Pratt en 1948 estimaron la incidencia sobre la base de cálculos teóricos multiplicándola de embarazo ectópico $(0.37 \%)$ por la de gemelos fraternos $(0.8 \%)$ originando una incidencia para embarazo heterotópico de $0.003 \%$ o aproximadamente 1:30.000. Sin embargo lo anterior asume una

\footnotetext{
* Profesor Asociado. Departamento de Ginecología y Obstetricia. Facultad de Salud. Universidad del Valle. Cali, Colombia.

** Residente rotante. Universidad del Cauca. Popayán, Colombia.
}

Figura 1.

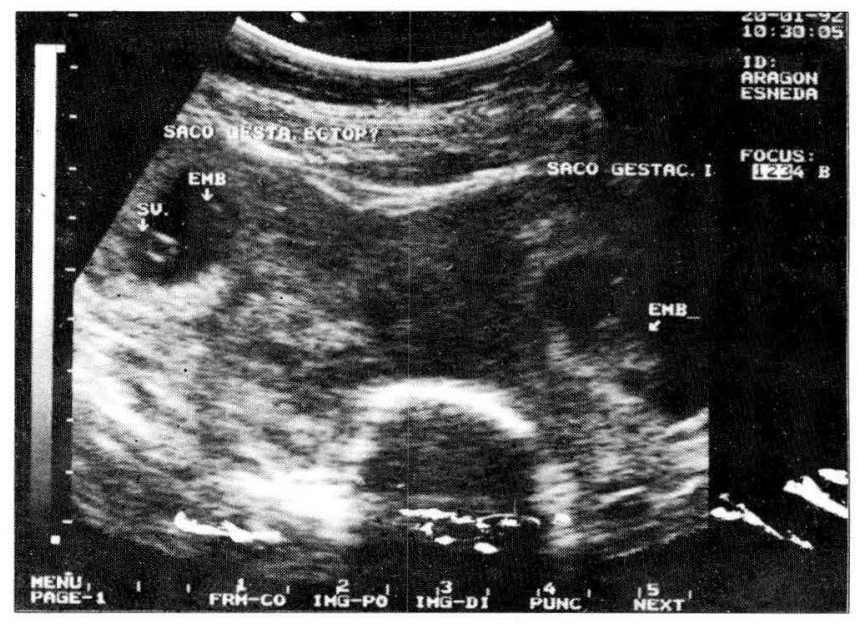

Informe ecográfico

Se observa útero en anteversoflexión, de bordes regulares, en su interior y a nivel de fondo se observa saco gestacional bien delimitado de bordes regulares, con una placa coriónica homogénea; en el interior de este saco hay feto único vivo que presenta actividad cardíaca que mide en su longitud céfalo caudal $20 \mathrm{~mm}$ que están en percentil promedio para embarazo de 9 sem., también se observa en este saco gestacional otras estructuras embionarias como el saco vitelino. También volumen de líquido amniótico normal.

Hacia el fondo del saco derecho y llegando hasta el cuerno derecho pero observándose fuera de éste se identifica formación irregular, ecomixta y también distalmente se observa saco gestacional de bordes regulares, bien delimitado, en el interior de este saco también se observa feto único vivo con actividad cardíaca el cual mide en su longitud céfalo caudal $12 \mathrm{~mm}$ que está en percentil promedio para un embarazo de $8 \mathrm{sem}$., también se observa en este saco líquido amniótico normal y saco vitelino de tamaño normal. No hay líquido en fondo de saco posterior.

frecuencia de embarazo ectópico constante lo que puede no ser cierto como lo muestran los nuevos reportes.

Aunque la etiología precisa de esa entidad no se conoce exactamente, se han identificado varios factores: (7).

- Enfermedad Pélvica Inflamatoria

- Embarazo Gemelar Dizigótico

- Uso de inductores de ovulación

- Técnicas modernas de reproducción asistida.

La superfecundación y superfetación han sido sospechadas pero sin confirmarse.

Las características clínicas presentan un espectro variable, una revisión de casos reportados muestran los siguientes síntomas comúnmente presentes: (7).

1. Dolor Abdominal 2. Masa Anexial 3. Irritación Peritoneal 4. Aumento de tamaño uterino.

Para muchos autores la ausencia de sangrado vaginal en un paciente con sospecha de embarazo ectópico es altamente sugestivo de gestación heterotópica; otros autores no han encontrado tal relación. (4, 8-10).

El diagnóstico preoperatorio se hace únicamente en un $10 \%(11)$. 
El tratamiento de esta entidad se fundamenta en la terminación del embarazo ectópico para evitar el riesgo de ruptura y hemorragia intraperitoneal.

Se han reportado 13 casos (12) en los cuales ambos embarazos han llegado a término con sobrevida de los productos durante el período neonatal. Sin embargo la literatura muestra una incidencia mayor de malformaciones congénitas y retardo mental en tales embarazos (13).

La mortalidad estimada oscila entre $20 \%$ a un $70 \%$ para el embarazo intrauterino y del $90 \%$ a $95 \%$ para el embarazo extrauterino $(8,13-14)$.
La mortalidad materna ha disminuido notablemente del 19\% en: 1935 a menos del $1 \%$ en la actualidad (12-13).

El embarazo heterotópico entraña un riesgo fetal y materno. De allí que un alto índice de sospecha en casos atípicos de gestación múltiple, embarazo ectópico y aborto sea esencial para un diagnóstico preoperatorio correcto.

Los hallazgos sugieren un embarazo intrauterino normal y otra posibilidad aunque es rara de 1 a 30 mil RN sería de un segundo embarazo ectópico no roto pero se debe descartar la posibilidad de un segundo embarazo en un cuerno de un útero bicorne por lo cual se sugiere laparoscopia.

\section{BIBLIOGRAFIA}

1. De Voe R.W., Pratt JH. Simultaneous intra and extrauterine pregnancy. Am. J. Obstet. Gynecol. 1948; 56: 1119.

2. Winer AE., Bergman WD., Fields CF. Combined intra and extrauterine pregnancy. Am. J. Obstet. Gynecol. 1957; 74: 170.

3. Hann LE., Bachman DM., McArdle C. Coexistent intrauterine and ectopic pregnancy: a re-evaluation. Radiology. 1984; 152: 151.

4. Falo PA., Solano S. and O' Regan M. Combined pregnancy, Va. Med. 1979; 106: 666 .

5. Richards SR., Stempel LE., Carlton BD. Heterotopic pregnancy: Reappraisal of incidence. Am. J. Obstet. Gynecol. 1982; 142: 928.

6. Dimitry ES. et al. Nine cases of heterotopic pregnancies in 4 years of in vitro fertilization. Fertil Steril 1990; 53: 107.

7. Winer AE., Bergman WD., Fields CF. Combined intra and extrauterine pregnancy. Am. J. Obstet. Gynecol. 1961; 24: 140.
8. Reece EA., Petrie RH., Sirmans ME., Finster M. Tood WD. Combined Intrauterine and extrauterine gestations: a review. Am. J. Obstet. Gynecol. 1983; 146: 323.

9. Brody S. and Stevens FL. Combined intra in extrauterine pregnancy, Obstet. Gynecol. 1963; 21: 129.

10. Ghandi J. Simultaneous intrauetrine and extrauterine pregnancies N.Y. State J. Med. 1967; 77: 1765.

11. Madhany N.H. Combined intra-and extrauterine (Tubal) pregnancies in two patients, East Afr. Med. J. 1977; 54: 505.

12. Felbo M. and Fenger HJ. Combined extra-and intrauterine pregnancy carried to term, Acta Obstet. Gynecol. Scand. 1966; 45: 140.

13. Hassim AM. Heterotopic pregnancy: Report of two cases, Cent. Afr. J. Med. 1966; 12: 181.

14. Sinnathuray TA. and Choo HT. Simultaneous intra and extrauterine pregnancy. A compresive report, Med. J. Malaya 1966; 21 : 53. 